\title{
Comparative analysis between 64- and 320-slice spiral computed tomography in the display of coronary artery stents and diagnosis of in-stent restenosis
}

\author{
JUNYAN YUE, JIE CHEN, WENGUANG DOU, YING HU, QIANG LI, FENGMEI ZHOU, \\ HONGKAI CUI, QINGWU WU and RUIMIN YANG
}

Imaging Center, The First Affiliated Hospital of Xinxiang Medical University, Weihui, Henan 453100, P.R. China

Received September 4, 2014; Accepted August 26, 2015

DOI: $10.3892 / \mathrm{etm} .2015 .2768$

\begin{abstract}
The aim of the present study was to compare the accuracy of 64-multi-slice spiral computed tomography (64-MSCT) and 320-MSCT in the display of coronary artery stents and diagnosis of in-stent restenosis. The data collected from the 64- and 320-MSCT coronary angiography of 93 patients following coronary artery stent implantation were retrospectively analyzed. The 64-MSCT group comprised 30 cases with 57 stents and the 320 -MSCT group comprised 63 cases with 93 stents. The image quality, heart rate of the patients and the radiation effective dose (ED) they were subjected to, were compared. Furthermore, the diagnostic abilities of 64-and 320-MSCT coronary angiography for in-stent restenosis were evaluated using invasive coronary angiography results as the gold standards. Statistically significant differences were observed in the heart rate and $\mathrm{ED}$ of the patients from the two groups $(\mathrm{P}<0.05)$, but no significant difference was identified in the accuracy index $(\mathrm{P}>0.05)$. The sensitivity, specificity, positive and negative predictive value and accuracy of the 64-MSCT group were found to be $100 \%$ (7/7), 93.94\% (31/33), $77.78 \%$ (7/9), 100\% (31/31) and 95\% (38/40), respectively, and those in the 320-MSCT group were found to be $100 \%$ (16/16), $95.89 \%$ (70/73), $84.21 \%$ (16/19), $100 \%(70 / 70)$ and $96.63 \%(86 / 89)$, respectively. The present findings suggest that both 64-MSCT and 320-MSCT can be used for follow-up and curative effect evaluation following coronary stent implantation; however, 320-MSCT has fewer requirements of the patients' heart rate and uses a lower radiation dose.
\end{abstract}

Correspondence to: Dr Ruimin Yang, Imaging Center, The First Affiliated Hospital of Xinxiang Medical University, 88 Jiankang Road, Weihui, Henan 453100, P.R. China

E-mail: ruiminyangdoc@126.com

Key words: coronary vessels, stents, tomography, computed tomography, in-stent restenosis

\section{Introduction}

The rise in the prevalence of coronary arterial obstructive disease and the increase in indicators of percutaneous coronary arterial stent implantation, have made the accurate detection of coronary arterial in-stent restenosis (ISR) using non-invasive techniques a focal point of research, and a positive conclusion has been drawn that multi-slice spiral computed tomography (MSCT) can be used to follow up the occurrence of coronary arterial ISR (1-7). From 2003 onwards, 64-MSCT has been used to detect coronary arterial ISR in assessable stents, and a relatively high specificity $(88-100 \%)$ and negative predictive value (NPV; $90-100 \%$ ) have been obtained $(6,8-14)$, due to its higher spatial and temporal resolution, compared with previous generations of MSCT. More recently, 320-MSCT systems able to achieve up to $16-\mathrm{cm}$ volumetric coverage in a single gantry rotation have become available (15). These systems simultaneously acquire 320 slices per rotation, and use a volumetric computed tomography (CT) data acquisition approach, thereby reducing contrast load, time of breath-hold and radiation (15). The high diagnostic performance of 320-slice CT coronary angiography (CTCA) in the assessment of coronary artery disease has been previously reported (16-18); however, the comparative analysis of the effectiveness of 64 and 320-MSCT in the detection of coronary artery stents and diagnosis of in-stent restenosis is lacking.

In the present study, data collected from the 64- and 320 -MSCT coronary angiography of 93 patients following coronary artery stent implantation at the First Affiliated Hospital of Xinxiang Medical University (Weihui, China) between December 2008 and June 2013 were analyzed. The patients included 30 cases with 51 stents that were subjected to $64-\mathrm{MSCT}$, and 63 cases with 99 stents that were subjected to 320 -MSCT. The aim of the study was to compare the diagnostic value of 64- and 320-MSCT in the display of coronary artery stents and the diagnosis of in-stent restenosis.

\section{Materials and methods}

Ethical approval and patient consent. The present retrospective study was approved by the Institutional Review Board of the First Affiliated Hospital of Xinxiang Medical University, 
and informed consent was waived by the institutional review board.

Patient data. A total of 93 patients who had coronary stents that had been in place for 30 days to 12 years were subjected to 64 or 320-slice CTCA. Of the 93 patients, 30 underwent 64 -slice CTCA [64-MSCT group; 27 men and 3 women (age range, $39-82$ years; mean age, $58.30 \pm 14.35$ years; body mass index (BMI), $\left.\left.23.40 \pm 1.56 \mathrm{~kg} / \mathrm{m}^{2}\right)\right]$ and 63 underwent 320-slice CTCA [320-MSCT group; 60 men and 3 women (age range, $45-86$ years; mean age, $59.40 \pm 14.00$ years; BMI, $24.10 \pm 1.53 \mathrm{~kg} / \mathrm{m}^{2}$ ).

Clinical data. The 64-MSCT group received 51 stents (1 stent in 15 cases, 2 stents in 9 cases and 3 stents in 6 cases). The 51 stents comprised 20 stainless steel coronary and 31 nitinol coronary stents. The 320-MSCT group received 99 stents ( 1 stent in 33 cases, 2 stents in 24 cases and 3 stents in 6 cases). The 99 stents comprised 21 stainless steel coronary and 78 nitinol coronary stents. No hypersensitivity to iodinated contrast media, renal insufficiency or any other contraindications were observed in any of the cases. No cases of arrhythmia or atrial fibrillation (AF) were observed in the 64-MSCT group; however, in the 320-MSCT group, 15 cases of arrhythmia or AF were identified.

CTCA data acquisition. In the 64-MSCT group, CTCA investigations were performed using 64-MSCT (Aquilion; Toshiba Medical Systems, Otawara, Japan) with 64 detector rows, each $0.5-\mathrm{mm}$ wide, and a gantry rotation time of $0.40 \mathrm{sec}$. If the heart rate of the patient exceeded 65 beats $/ \mathrm{min}$, oral $\beta$-blocking medication (25-75 mg propranolol hydrochloride tablets) was administered $1 \mathrm{~h}$ before examination, unless contraindicated. A triphasic protocol was used for the intravenous administration of the contrast medium. The total volume of the nonionic contrast agent (Ultravist ${ }^{\circledR} 370$ injection; Bayer Schering Pharma AG, Leverkusen, Germany) injected into the median cubital vein was $60-85 \mathrm{ml}$ (volume based on body weight: $\leq 50 \mathrm{~kg}, 60 \mathrm{ml} ; 50-60 \mathrm{~kg}, 70 \mathrm{ml} ; 60-70 \mathrm{~kg}, 80 \mathrm{ml}$; and $>70 \mathrm{~kg}, 85 \mathrm{ml}$ ) at a flow rate of $5.0-5.5 \mathrm{ml} / \mathrm{sec}$, followed by $40 \mathrm{ml}$ normal saline (NS). Following the 6-sec injection of the contrast agent, an automated peak enhancement detection technique was used to record the arrival of the contrast agent in the ascending aorta, in order to synchronize the arrival of the contrast media and the CT data acquisition, using a threshold of +180 Hounsfield units. CTCA images were acquired during an inspiratory breath-hold of $\sim 8 \mathrm{sec}$ covering an entire R-R interval of the retrospective electrocardiogram (ECG)-gating.

In the 320-MSCT group, CTCA investigations were performed using 320-MSCT (Aquilion ONE; Toshiba Medical Systems) with 320 detector rows, each $0.5-\mathrm{mm}$ wide, and a gantry rotation time of $0.35 \mathrm{sec}$. If the heart rate of the patient exceeded 70 beats/min, oral $\beta$-blocking medication $(25-75 \mathrm{mg}$ propranolol hydrochloride tablets) was administered $1 \mathrm{~h}$ before examination, unless contraindicated. A triphasic protocol was used for the intravenous administration of the contrast medium. The total volume of the nonionic contrast agent (Ultravist ${ }^{\circledR} 370$ injection; Bayer Schering Pharma AG) injected into the median cubital vein was 50-70 ml (volume based on BMI: $\leq 20$, $50 \mathrm{ml}$; 20-25, 50-60 ml; 25-30, 60-70 ml; and >30, $70 \mathrm{ml}$ ) at a flow rate of $5.0-5.5 \mathrm{ml} / \mathrm{sec}$, followed by $40 \mathrm{ml} \mathrm{NS}$. Following the 6-sec injection of the contrast agent, an automated peak enhancement detection technique was used to determine the arrival of the contrast agent in the thoracic aorta, in order to synchronize the arrival of the contrast media and the CT data acquisition, using a threshold of +350 Hounsfield units. An inspiratory breath-hold following the 12-sec injection of the contrast agent was required for the acquisition of the CT data. During the CT data acquisition, the ECG was registered simultaneously for prospective triggering of data acquisition. The phase window was set at $30-80 \%$ of the R-R interval in patients with a stable heart rate $(\leq 70$ beats $/ \mathrm{min})$. In the patients with a heart rate $>70$ beats $/ \mathrm{min}$, CTCA data acquisition was performed during multiple heart beats (typically two).

In both groups, the entire heart was examined. Tube voltage and current were adapted to the BMI of the patient (tube voltage range, 110-140 kV; maximal tube current, 400-580 mA).

An initial data set was reconstructed at the optimal phase of the R-R interval (typically 75\%). A slice thickness of $0.50 \mathrm{~mm}$ was obtained and the reconstruction interval was set to $0.25 \mathrm{~mm}$. If multiple phases were obtained, the cardiac phase with the least motion artifacts was selected (19). With regard to processing and assessment, images were transferred to a remote workstation with dedicated CTCA analysis software (Vitrea 2.0 or FX 3.1.1.0; Vital Images, Minnetonka, MN, USA). During the CTCA examination the highest heart rates recorded were 73 beats/min in the 64-MSCT group and 88 beats/min in the $320-$ MSCT group.

CTCA data analysis. CTCA image analysis was performed by two observers in consensus, experienced in the evaluation of CTCA and blinded to the results of invasive coronary angiography (ICA). First, three-dimensional volume-rendered reconstructions were used to gather information regarding the anatomy and status of the coronary arteries. Secondly, axial slices were visually examined for significant narrowing, which was achieved by determining the presence of $\geq 50 \%$ reduction of the luminal diameter and vessel occlusion (20). Thirdly, the analysis was assisted by curved multiplanar reconstructions of all vessels.

Image quality assessments. Adequately reconstructed CTCA images of stented segments were visually classified into 4 grades as follows: Grade 1, visible stent and stent lumen without metal artifacts; grade 2, visible stent and stent lumen with slight metal artifacts; grade 3, visible stent but invisible stent lumen with significant metal artifacts; grade 4, invisible stent and stent lumen with severe metal artifacts.

Significant ISR was diagnosed from the CTCA results if intraluminal low-attenuation filling defects narrowed the stent lumen diameter or the vessel lumen within $5 \mathrm{~mm}$ away from the stent by $>50 \%$.

Quantification of radiation dose. Radiation effective dose (ED) was quantified by a dose-length product conversion factor of $0.017 \mathrm{mSv} /(\mathrm{mGy} . \mathrm{cm})$. Following the examination, the CT machines (Aquilion and Aquilion ONE) provided a dose-length product automatically.

Statistical analysis. The results of ICA as a gold standard, and the sensitivity, specificity, positive predictive value (PPV), 
Table I. Validation of 64-slice CTCA compared with ICA for in-stent restenosis.

\begin{tabular}{lccr}
\hline & \multicolumn{2}{c}{ 64-Slice CTCA results } & \\
\cline { 2 - 3 } ICA results & Positive & Negative & Total \\
\hline Positive & 7 & 2 & 9 \\
Negative & 0 & 31 & 31 \\
Total & 7 & 33 & 40 \\
\hline
\end{tabular}

ICA, invasive coronary angiography; CTCA, computed tomography coronary angiography.

Table II. Validation of 320-slice CTCA compared with ICA for in-stent restenosis.

320-Slice CTCA results

\begin{tabular}{lccc} 
& Positive & Negative & Total \\
\cline { 2 - 3 } ICA results & 16 & 3 & 19 \\
Positive & 0 & 70 & 70 \\
Negative & 16 & 73 & 89 \\
Total & 16 & 73 \\
\hline
\end{tabular}

ICA, invasive coronary angiography; CTCA, computed tomography coronary angiography.

Table III. Comparative analysis of heart rate, BMI and ED between the two groups.

\begin{tabular}{lccc}
\hline Variable & Heart rate & BMI & ED \\
\hline 64-MSCT group & $65.10 \pm 4.78$ & $23.30 \pm 1.46$ & $20.00 \pm 3.26$ \\
320-MSCT group & $71.24 \pm 7.91$ & $24.06 \pm 1.51$ & $12.26 \pm 1.81$ \\
Z-score & 3.05 & 2.02 & 6.21 \\
P-value & 0.02 & 0.04 & 0.00 \\
\hline
\end{tabular}

Values are mean \pm standard deviation. MSCT, multi-slice spiral computed tomography; BMI, body mass index; ED, effective dose.

negative predictive value (NPV) and accuracy of the two MSCT methods for the detection of significant ISR in CTCA were calculated for assessable stents. Continuous data are expressed as mean \pm standard deviation. The $\chi^{2}$, Fisher's exact and rank-sum tests were used to compare differences between the two groups. $\mathrm{P}<0.05$ was considered to indicate a statistically significant difference and all reported P-values were two-sided. Statistical analyses were performed using SPSS software, version 13 (SPSS, Inc., Chicago, IL, USA).

\section{Results}

Results of image quality assessment. In the 64-MSCT group, the images of the 20 stainless steel coronary stents comprised
9 images classified as grade 3 and 11 as grade 2, and for the 31 nitinol coronary stents, 29 of the images were classified as grade 1, and 2 as grade 2. In the 320 -MSCT group, the images of the 21 stainless steel coronary stents comprised 10 images classified as grade 3 and 11 classified as grade 2, and for the 78 nitinol coronary stents, 74 were classified as grade 1 , and 4 as grade 2 . No statistically significant differences were observed in the image quality assessments between the 64- and 320-MSCT groups for stainless steel $\left(\chi^{2}=0.028\right)$ and nitinol coronary $\left(\chi^{2}=0.075\right)$ stents $(\mathrm{P}>0.05)$.

Differences of sensitivity, specificity, $P P V, N P V$ and accuracy between 64-MSCT and 320-MSCT for significant ISR. Images of stents classified as grade 3 or 4 were excluded from the imaging quality assessments. In the 64-MSCT group, 40 stents were classified as assessable, and 9 stents in 8 patients were diagnosed by CTCA to have significant ISR; however, 7 of 9 stents in 6 patients were found by ICA to have significant ISR (Fig. 1). These stents had been in place for 6 months to 4-years. The sensitivity, specificity, PPV, NPV and accuracy of 64-MSCT for significant ISR in all assessable stents were $100 \%$ (7/7), 93.94\% (31/33), 77.78\% (7/9), 100\% (31/31) and 95\% (38/40), respectively (Table I). In the 320-MSCT group, 89 stents were classified as assessable, and 19 stents in 88 patients were diagnosed by CTCA to have significant ISR; however, 16 of 19 stents in 15 patients were found by ICA to have significant ISR (Fig. 2). These stents had been in place for 2 months to 7 years. The sensitivity, specificity, PPV, NPV and accuracy of 320-MSCT for significant ISR in all assessable stents were $100 \%$ (16/16), 95.89\% (70/73), 84.21\% (16/19), $100 \%(70 / 70)$ and $96.63 \%(86 / 89)$, respectively (Table II). No statistically significant difference was identified in the sensitivity, specificity, PPV, NPV and accuracy between the 64-MSCT and 320-MSCT groups tested using Fisher's exact test $(\mathrm{P}>0.05)$.

Differences of heart rate and ED between 64-MSCT and 320-MSCT. Statistically significant differences were observed in the heart rate and ED of the patients between the 64-MSCT and 320-MSCT groups $(\mathrm{P}<0.05)$ (Table III).

\section{Discussion}

ISR is a major long-term complication of percutaneous coronary treatment and is mainly due to neointimal proliferation, but also mechanical causes (6). Generally, if the vessel lumen on both sides of the stent does not narrow and the density in the stent is the same as the density of adjacent normal vessels, this is considered a direct sign of lack of ISR. An indirect sign of an absence of ISR is a well-filled distant vessel of the stent; however, if the stent has been distorted, the vessel distant from of the stent exhibits a filling defect, narrowing or intermittent display (21). It has been shown that $20-30 \%$ of patients develop ISR at $\sim 6$ months after coronary stent implantation (22). Although the results of the present study indicate that patients develop ISR $>6$ months after the coronary stent implantation, that may be due to the relatively small number of patients included in the study.

The diagnosis of ISR has been a subject of particular interest to cardiologists and radiologists $(3,5,7,17,23-31)$. The 

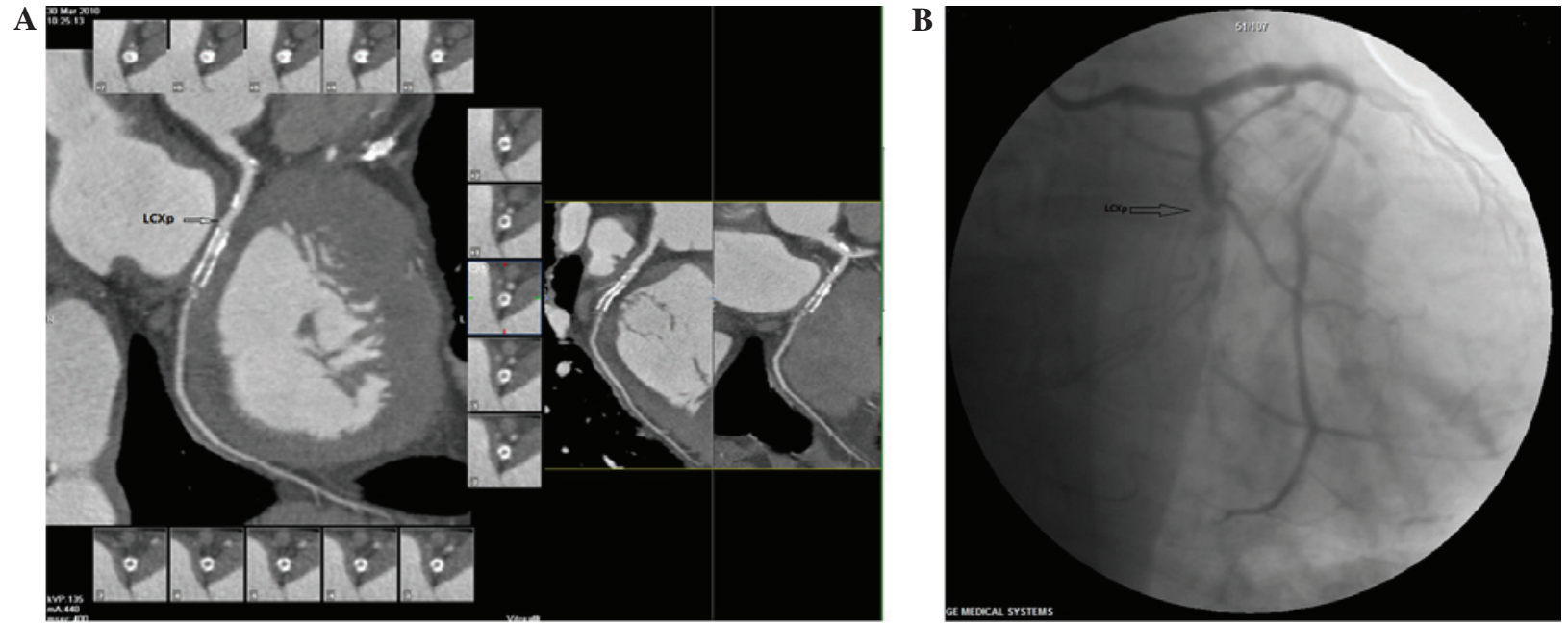

Figure 1. An example of significant in-stent restenosis of the proximal region of the left cirumflex artery (LCXp) using 64-slice CTCA and confirmed on ICA. A subtotal occlusion in the stent of LCXp was identified on CTCA. (A) Curved multiplanar reconstruction depicting a subtotal occlusion in the stent of LCXp. (B) The finding was confirmed on ICA (arrow). ICA, invasive coronary angiography; CTCA, computed tomography coronary angiography.
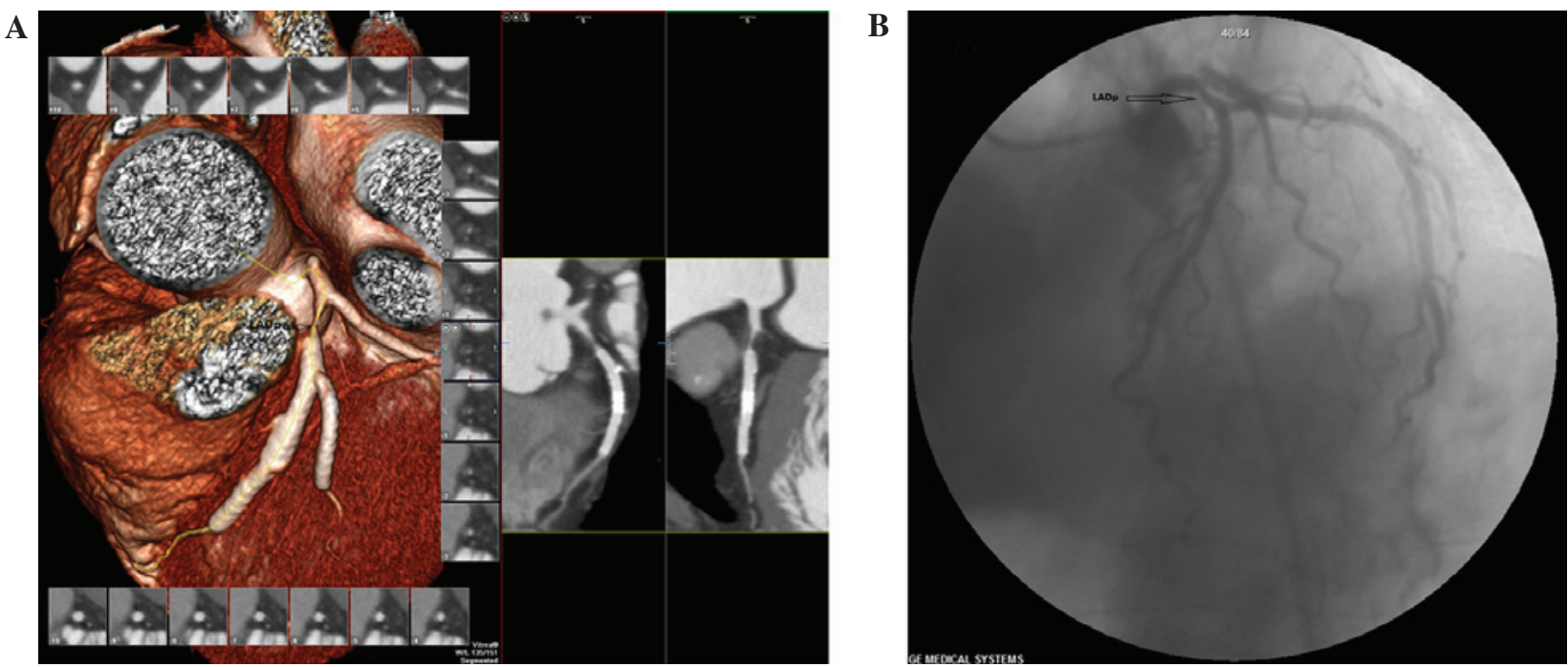

Figure 2. An example of a significant ISR in the proximal region of the left anterior descending artery using 320-slice CTCA and confirmed on ICA. A severe stenosis in the proximal vessel of stent was identified on CTCA. (A) Curved multiplanar reconstruction depicting a severe stenosis in the proximal vessel of stent. (B) The finding was confirmed on ICA (arrow). ICA, invasive coronary angiography; CTCA, computed tomography coronary angiography.

main finding of the present study was the lack of statistically significant differences in the sensitivity, specificity, PPV, NPV and accuracy between 64- and 320-MSCT in the diagnosis of significant ISR $(P>0.05)$, suggesting that, when the number of detector rows of spiral CT reaches a certain value, if not to improve the spatial resolution of $\mathrm{CT}$, but only to increase the number of detector rows, no further effective improvements in the quality of CT imaging can be achieved., with any further increases in the number of detector rows of spiral CT not causing any improvement of the spatial resolution of CT. In addition, the present results indicated that both 64- and 320-MSCT failed to display the stainless steel coronary stents clearly, and that the grade of image quality assessments for these stents was lower than that of the nitinol coronary stents. This finding also suggests that stent material plays a central role in the follow-up and curative effect evaluation following stent implantation (2).
It may be concluded that 64-MSCT remains underdeveloped and inferior to 320-MSCT in all aspects. First, 64-MSCT scanning is easily affected by the patient's condition and requires a regular heartbeat, a low heart rate and lack of AF in order for it to be successful. Obtaining an acceptable image when the patient exhibits an irregular heartbeat, high heart rate and AF can be challenging. The results demonstrated that the mean heart rate of the 64-MSCT group was significantly lower than that of the 302-MSCT group $(\mathrm{P}=0.02)$. This indicates that there is a greater restriction on the patient's heart rate in 64-slice CTCA than in 320-slice CTCA. Secondly, the ED received by the patients in the 64-MSCT group was higher than that in the 320-MSCT group. A previous study has reported that the use of 320- instead of 64-MSCT scanning could signify a considerable reduction in the ED received by patients (17). The aforementioned finding is not only associated with the differences between 64- and 320-MSCT scans, 
but also with other factors that affect the scanning procedure, such as the scan mode and reconstruction methods used during the examination. The present study also showed that the ED of the patients in the 320-MSCT group was lower than that of the patients in the 64-MSCT group $(\mathrm{P}<0.01)$, a finding that was fairly consistent with results reported in the literature $(15,17)$.

The present study did, however, have certain limitations: First, the number of samples was small, and therefore further studies with a larger sample should be conducted. Secondly, the effect of the stent material on image quality could not be observed and thirdly, the sign of ISR has not been described in detail.

In conclusion, both 64- and 320-MSCT are suitable for use in follow-up and curative effect evaluation following coronary stent implantation; however, 320-MSCT is less restricted by the patient's heart rate and uses a lower dose of radiation.

\section{Acknowledgements}

This study was supported by the Health Department of Henan Province, by a grant to the research project entitled 'The applied research of 320-slice spiral CT used in coronary arterial in-stent restenosis' (no. 201003078). The authors would also like to thank the doctors, technicians and nurses of the Departments of DSA and Radiology of the Affiliated Hospital of Xinxiang Medical University for their assistance in case collection.

\section{References}

1. Ehara M, Kawai M, Surmely JF, Matsubara T, Terashima M, Tsuchikane E, Kinoshita Y, Ito T, Takeda Y, Nasu K, et al: Diagnostic accuracy of coronary in-stent restenosis using 64-slice computed tomography: Comparison with invasive coronary angiography. J Am Coll Cardiol 49: 951-959, 2007.

2. Chung SH, Kim YJ, Hur J, Lee HJ, Choe KO, Kim TH and Choi BW: Evaluation of coronary artery in-stent restenosis by 64-section computed tomography: Factors affecting assessment and accurate diagnosis. J Thorac Imaging 25: 57-63, 2010.

3. Hong C, Chrysant GS, Woodard PK and Bae KT: Coronary artery stent patency assessed with in-stent contrast enhancement measured at multi-detector row CT angiography: Initial experience. Radiology 233: 286-291, 2004.

4. Gaspar T, Halon DA, Lewis BS, Adawi S, Schliamser JE, Rubinshtein R, Flugelman MY and Peled N: Diagnosis of coronary in-stent restenosis with multidetector row spiral computed tomography. J Am Coll Cardiol 46: 1573-1579, 2005.

5. Gilard M, Cornily JC, Pennec PY, Le Gal G, Nonent M, Mansourati J, Blanc JJ and Boschat J: Assessment of coronary artery stents by 16 slice computed tomography. Heart 92: 58-61, 2006.

6. Ehara M, Surmely JF, Kawai M, Katoh O, Matsubara T, Terashima M, Tsuchikane E, Kinoshita Y, Suzuki T, Ito T, et al: Diagnostic accuracy of 64-slice computed tomography for detecting angiographically significant coronary artery stenosis in an unselected consecutive patient population: Comparison with conventional invasive angiography. Circ J 70: 564-571, 2006.

7. Seifarth H, Ozgün M, Raupach R, Flohr T, Heindel W, Fischbach R and Maintz D: 64-Versus 16-slice CT angiography for coronary artery stent assessment: In vitro experience. Invest Radiol 41: 22-27, 2006.

8. Rist C, von Ziegler F, Nikolaou K, Kirchin MA, Wintersperger BJ, Johnson TR, Knez A, Leber AW, Reiser MF and Becker CR: Assessment of coronary artery stent patency and restenosis using 64-slice computed tomography. Acad Radiol 13: 1465-1473, 2006.

9. Rixe J, Achenbach S, Ropers D, Baum U, Kuettner A, Ropers U, Bautz W, Daniel WG and Anders K: Assessment of coronary artery stent restenosis by 64 -slice multi-detector computed tomography. Eur Heart J 27: 2567-2572, 2006.
10. Oncel D, Oncel G and Karaca M: Coronary stent patency and in-stent restenosis: Determination with 64-section multidetector CT coronary angiography-initial experience. Radiology 242: 403-409, 2007.

11. Carrabba N, Bamoshmoosh M, Carusi LM, Parodi G, Valenti R, Migliorini A, Fanfani F and Antoniucci D: Usefulness of 64-slice multidetector computed tomography for detecting drug eluting in-stent restenosis. Am J Cardiol 100: 1754-1758, 2007.

12. Das KM, El-Menyar AA, Salam AM, Singh R, Dabdoob WA, Albinali HA and Al Suwaidi J: Contrast-enhanced 64-section coronary multidetector CT angiography versus conventional coronary angiography for stent assessment. Radiology 245: 424-432, 2007.

13. Schuijf JD, Pundziute G, Jukema JW, Lamb HJ, Tuinenburg JC, van der Hoeven BL, de Roos A, Reiber JH, van der Wall EE, Schalij MJ and Bax JJ: Evaluation of patients with previous coronary stent implantation with 64-section CT. Radiology 245: 416-423, 2007.

14. Tedeschi C, Ratti G, De Rosa R, Sacco M, Borrelli F, Tammaro P, Covino G, Montemarano E, Cademartiri F, Runza G, et al: Usefulness of multislice computed tomography to assess patency of coronary artery stents versus conventional coronary angiography. J Cardiovasc Med (Hagerstown) 9: 485-492, 2008.

15. Hein PA, May J, Rogalla P, Butler C, Hamm B and Lembcke A: Feasibility of contrast material volume reduction in coronary artery imaging using 320-slice volume CT. Eur Radiol 20: 1337-1343, 2010.

16. de Graaf FR, Schuijf JD, van Velzen JE, Kroft LJ, de Roos A, Reiber JH, Boersma E, Schalij MJ, Spanó F, Jukema JW, et al: Diagnostic accuracy of 320-row multidetector computed tomography coronary angiography in the non-invasive evaluation of significant coronary artery disease. Eur Heart J 31: 1908-1915, 2010.

17. Dewey M,Zimmermann E, Deissenrieder F, Laule M, Dübel HP, Schlattmann P, Knebel F, Rutsch W and Hamm B: Noninvasive coronary angiography by 320 -row computed tomography with lower radiation exposure and maintained diagnostic accuracy: Comparison of results with cardiac catheterization in a head-to-head pilot investigation. Circulation 120: 867-875, 2009.

18. de Graaf FR, Schuijf JD, van Velzen JE, Boogers MJ, Kroft LJ, de Roos A, Reiber JH, Sieders A, Spanó F, Jukema JW, et al: Diagnostic accuracy of 320-row multidetector computed tomography coronary angiography to noninvasively assess in-stent restenosis. Invest Radiol 45: 331-340, 2010.

19. Desbiolles L, Leschka S, Plass A, Scheffel H, Husmann L, Gaemperli O, Garzoli E, Marincek B, Kaufmann PA and Alkadhi $\mathrm{H}$ : Evaluation of temporal windows for coronary artery bypass graft imaging with 64-slice CT. Eur Radiol 17: 2819-2828, 2007.

20. Raff GL, Abidov A, Achenbach S, Berman DS, Boxt LM, Budoff MJ, Cheng V, DeFrance T, Hellinger JC and Karlsberg RP; Society of Cardiovascular Computed Tomography: SCCT guidelines for the interpretation and reporting of coronary computed tomographic angiography. J Cardiovasc Comput Tomogr 3: 122-136, 2009.

21. Schmitt F, Grosu D, Mohr C, Purdy D, Salem K, Scott KT and Stoeckel B: 3 Tesla MRI: Successful results with higher field strengths. Radiologe 44: 31-47, 2004.

22. Peng LJ and Tian GP: Inflammatory reaction and stent restenosis after coronary stent implantation. Shi Yong Xin Nao Fei Xue Guan Bing Za Zhi 14: 13-15, 2006 (In Chinese).

23. Kitagawa T, Fujii T, Tomohiro Y, Maeda K, Kobayashi M, Kunita E and Sekiguchi Y: Noninvasive assessment of coronary stents in patients by 16 -slice computed tomography. Int J Cardiol 109: 188-194, 2006.

24. Zhu H, Warner JJ, Gehrig TR and Friedman MH: Comparison of coronary artery dynamics pre- and post-stenting. J Biomech 36: 689-697, 2003.

25. Şatiroğlu Ö, Bostan M and Bozkur E: Acute coronary syndrome due to bare metal stent fracture in the right coronary artery. Kardiol Pol 69: 859-861, 2011.

26. Bilen E, Saatci Yasar A, Bilge M, Karakas F, Kirbas O and Ipek G: Acute coronary syndrome due to complete bare metal stent fracture in the right coronary artery. Int J Cardiol 139: e44-e46, 2010.

27. Kang WC, Han SH, Ahn TH and Shin EK: Acute myocardial infarction caused by rupture of minimal intrastent intimal hyperplasia after implantation of bare-metal stent. Int J Cardiol 120: e37-e40, 2007. 
28. Giuliani G, Maehara A and Parodi G: Vessel response evaluation by optical coherence tomography after drug-eluting stent implantation in the left main coronary artery. G Ital Cardiol (Rome) 11: 246, 2010 (In Italian).

29. Ruygrok PN, Webster MW, Ardill JJ, Chan CC, Mak KH, Meredith IT, Stewart JT, Ormiston JA and Price S: Vessel caliber and restenosis: A prospective clinical and angiographic study of NIR stent deployment in small and large coronary arteries in the same patient. Catheter Cardiovasc Interv 59: 165-171, 2003.
30. Sousa JE, Costa MA, Sousa AG, Abizaid AC, Seixas AC, Abizaid AS, Feres F, Mattos LA, Falotico R, Jaeger J, et al: Two-year angiographic and intravascular ultrasound follow-up after implantation of sirolimus-eluting stents in human coronary arteries. Circulation 107: 381-383, 2003.

31. Ahmed JM: Serial intravascular ultrasound assessment of the efficacy of intracoronary gamma radiation for preventing recurrent in-stent restenosis. Minerva Cardioangiol 50: 507-515, 2002. 